disordered breathing versus alcohol-impaired controls. Laryngoscope 1999;109:1648-54.

7. Roehrs T, Burduvali E, Bonahoom A, Drake C, Roth T. Ethanol and sleep loss: a "dose" comparison of impairing effects. Sleep 2003;26:981-5.

DOI:10.1503/cmaj.1040701

\section{TB and adrenal insufficiency}

$\mathrm{R}_{\mathrm{s}}$ onik Kanani and Aleixo Muise ${ }^{1}$ describe a case of intra-abdominal peritonitis associated with abdominal pain and hypotension secondary to intra-abdominal tuberculosis (TB). The clinical presentation in this case is suspicious for adrenal insufficiency.

$\mathrm{TB}$ is one of the leading causes of adrenal insufficiency worldwide, but the presence of TB may be difficult to diagnose. In a large retrospective study of autopsy results in Hong Kong, Lam and $\mathrm{Lo}^{2}$ found active TB in $6.5 \%$ of cases; however, in more than $70 \%$ of these patients, TB was diagnosed only at autopsy. Adrenal insufficiency was found in $6 \%$ of the patients with active TB.

It may also be difficult to identify $\mathrm{TB}$ as the cause of adrenal insufficiency, as illustrated by Serter and associates. ${ }^{3}$
These authors described a 61-year-old man with adrenal insufficiency and an adrenal mass. The results of tuberculin skin testing, staining for acid-fast bacilli and culture were all negative; only histologic examination after adrenalectomy confirmed the diagnosis of TB.

In patients presenting with shock and risk factors for TB it is important to maintain a high index of suspicion for adrenal insufficiency. When in doubt, administration of a stress dose of steroids while awaiting the results of corticotropin (ACTH) and cortisol measurement may save the patient's life.

\section{Wael M.R. Haddara \\ Stan H.M. van Uum \\ Division of Endocrinology and \\ Metabolism \\ University of Western Ontario \\ London, Ont.}

\section{References}

1. Kanani R, Muise A. A 17-year-old male with an unusual case of peritonitis. CMAF 2004;170(10): 1541.

2. Lam KY, Lo CY. A critical examination of adrenal tuberculosis and a 28-year autopsy experience of active tuberculosis. Clin Endocrinol (Oxf.) 2001;54(5):633-9.

3. Serter R, Koc G, Demirbas B, Culha C, On- goren $\mathrm{AU}$, Ustun $\mathrm{H}$, et al. Acute adrenal crisis together with unilateral adrenal mass caused by isolated tuberculosis of adrenal gland. Endocr Pract 2003;9(2):157-61.

DOI:10.1503/cmaj.1041046

\section{[The authors respond:]}

A lthough TB is one of the leading A causes of adrenal insufficiency in the world and should be considered in patients presenting with shock and a history suggestive of $\mathrm{TB},{ }^{1}$ the index of suspicion for adrenal insufficiency was quite low in the case that we reported. ${ }^{2}$ Although the patient was initially hypotensive, his blood pressure did respond to fluid administration, and his electrolyte levels were initially normal. In contrast, patients in adrenal crisis usually require steroid treatment and do not respond readily to fluids. Furthermore, the patient underwent multiple CT examinations of the abdomen, all of which showed that the adrenal glands were normal. We did not check the patient's cortisol level, nor did we do corticotropin stimulation test, as it was extremely unlikely that the patient had 